\title{
Differential Space-Frequency Modulation via Smooth Logical Channel for Broadband Wireless Communications
}

\author{
Weifeng Su, Member, IEEE, and K. J. Ray Liu, Fellow, IEEE
}

\begin{abstract}
In this letter, a differential space-frequency modulation (DSFM) scheme is proposed for multiple input multiple-output (MIMO)-orthogonal frequency-division multiplexing (OFDM) systems in broadband wireless communications. We assume that the fading channels keep constant only within each OFDM block, and may change independently from one OFDM block to another. The differential schemes proposed for MIMO-OFDM systems in the literature cannot successfully decode with such a rapidly fading channel, since the successful decoding of the previously existing schemes relies on the assumption that the fading channel keeps constant within a period of several OFDM blocks, and it changes slowly from a period of several OFDM blocks to another. In our proposed DSFM scheme, the transmitted signals are differentially encoded in the frequency domain within each OFDM block. Thus, the differential decoding can be performed over subcarriers within each single OFDM block. Furthermore, if a statistical channel power-delay profile (PDP) is known at the transmitter, we propose to create a smooth logical channel to improve the performance of the DSFM scheme. We obtain the smooth logical channel by sorting the channel frequency responses over subcarriers from a statistical point of view. If the logical channel is not smooth enough, we further consider a pruning process in which we use only the "good" part of the channel and get rid of the "bad" part of the channel. Simulation results show that the proposed DSFM scheme over a smooth logical channel (with pruning, if necessary) performs well for various channel PDPs.
\end{abstract}

Index Terms-Differential space-time (ST) coding, frequency-selective fading channels, full diversity, multiple-input multiple-output (MIMO)-orthogonal frequency-division multiplexing (OFDM) systems, space-frequency coding.

\section{INTRODUCTION}

$\mathbf{M}$ ULTIPLE-INPUT multiple-output (MIMO) systems have shown great potential in the next generation wireless communications due to their promising huge capacity [1]. Among abundant space-time (ST) coding and modulation schemes developed for MIMO systems, differential space-time modulation (DSTM) schemes [2]-[4] have been proposed for the case of flat-fading channels, in which both the transmitter and the receiver do not need to know the exact channel state information (CSI). With such schemes, there is only a $3-\mathrm{dB}$

Paper approved by N. Al-Dhahir, the Editor for Space-Time, OFDM, and Equalization of the IEEE Communications Society. Manuscript received September 24, 2004; revised February 25, 2005 and June 2, 2005. This work was supported in part by the U.S. Army Research Laboratory under Cooperative Agreement DAAD 190120011. This paper was presented in part at the IEEE Global Telecommunications Conference, Dallas, TX, Nov. 29-Dec. 3, 2004.

W. Su is with the Department of Electrical Engineering, State University of New York at Buffalo, Buffalo, NY 14260 USA (e-mail: weifeng@ eng.buffalo.edu).

K. J. R. Liu is with the Department of Electrical and Computer Engineering and Institute for Systems Research, University of Maryland, College Park, MD 20742 USA (e-mail: kjrliu@eng.umd.edu).

Digital Object Identifier 10.1109/TCOMM.2005.860070 performance degradation, compared with the case of coherent detection in which perfect CSI should be known at the receiver. The avoiding of channel estimations and the comparable performances make the DSTM schemes attractive, as many DSTM signals have been designed since then [4]-[8].

In broadband wireless communications, the fading channel exhibits frequency selectivity due to the multiple delay paths that may result in severe intersymbol interference. Noncoherent MIMO systems with orthogonal frequency-division multiplexing (OFDM) have been investigated in [9] and [10], but not specific to a differential encoding/decoding strategy. In [11], two differential coding schemes were proposed for MIMO frequency-selective fading channels: one for a single-carrier scenario and the other for a multicarrier scenario with OFDM. However, both schemes exploited only the spatial diversity, regardless of the frequency diversity which is available in the fading channels. A differential encoding scheme that aims to exploit the frequency diversity was proposed in [12], but it works only for OFDM systems with a single transmit antenna. Later in [13]-[15], differential encoding/decoding schemes were established for MIMO-OFDM systems to achieve both the spatial and frequency diversities. The differential decoding depends on the assumption that the fading channel stays constant over a transmission period of $M_{t}$ (the number of transmit antennas considered in the systems) OFDM blocks. The differential schemes in [12]-[14] followed the DSTM approaches in [3] and [4] by using recursive differential encoding/decoding equations. The differential encoding/decoding was performed in the temporal dimension, i.e., the transmitted/received signals within a period of $M_{t}$ OFDM blocks depend on those in the previous $M_{t}$ OFDM blocks. These schemes also assumed that the fading channel keeps constant over $M_{t}$ OFDM blocks, and further assumed that the channel changes slowly from one period of $M_{t}$ OFDM blocks to another. A differential scheme in the frequency dimension was also proposed in [13], in which the differential encoding/decoding process was performed over adjacent subcarriers. However, this scheme also depends on the assumption that the fading channel is constant over $M_{t}$ OFDM blocks. In the case of a large number of transmit antennas, this assumption is not valid, even for a typical slow-fading scenario. Note that a comparison of frequency- and time-domain differential modulation has been studied in [16] for a specific 5.2-GHz wireless asynchronous transfer mode (ATM) system with a single transmit antenna.

In this letter, we propose a differential space-frequency modulation (DSFM) scheme for MIMO-OFDM systems with a "fast" fading scenario, in which the fading channel keeps constant only within each single OFDM block, and it may change independently from one OFDM block to another. The 
conventional differential schemes cannot successfully decode with such a rapidly fading channel. We differentially encode the transmitted signals in the frequency domain within each OFDM block. Thus, the differential decoding can be performed over subcarriers within each single OFDM block. Moreover, if a statistical channel power-delay profile (PDP) is known at the transmitter, we propose to create a smooth logical channel to improve the performance of the DSFM scheme. We obtain the smooth logical channel by sorting the channel-frequency responses over subcarriers by use of Dijkstra's algorithm [19], [20] from a statistical point of view.

\section{Channel Model AND DSFM Scheme}

We consider a MIMO-OFDM system with $M_{t}$ transmit and $M_{r}$ receive antennas. The OFDM has $N$ subcarriers. Both the transmitter and receiver do not know the CSI. The MIMO channel is assumed to be constant within each OFDM block period, and it may change independently from one OFDM block to another. Suppose that the fading channels between different transmit and receive antenna pairs have $L$ paths and the same PDP. In the frequency domain, the received signal at the $n$th $(0 \leq n \leq N-1)$ subcarrier at receive antenna $j$ can be written as

$$
y_{j}(n)=\sqrt{\rho} \sum_{i=1}^{M_{t}} x_{i}(n) H_{i, j}(n)+z_{j}(n)
$$

where $x_{i}(n)$ is the channel symbol transmitted over the $n$th subcarrier by transmit antenna $i, H_{i, j}(n)$ is the channel frequency response at the $n$th subcarrier between transmit antenna $i$ and receive antenna $j$, and $z_{j}(n)$ denotes the additive complex Gaussian noise with zero mean and unit variance at the $n$th subcarrier at receive antenna $j$. The channel frequency response $H_{i, j}(n)$ can be specified as $H_{i, j}(n)=\sum_{l=0}^{L-1} \alpha_{i, j}(l) e^{-\mathrm{j} 2 \pi n \Delta f \tau_{l}}$, where $\alpha_{i, j}(l)$ is the complex amplitude of the $l$ th path, $\tau_{l}$ is the delay of the $l$ th path, $\Delta f=1 / T$ is the subcarrier separation, $T$ is the OFDM symbol period, and $\mathbf{j}=\sqrt{-1}$. The $\alpha_{i, j}(l)$ 's are modeled as zero-mean, complex Gaussian random variables with variances $E\left|\alpha_{i, j}(l)\right|^{2}=\delta_{l}^{2}$, where $E$ stands for the expectation. The powers of the $L$ paths are normalized such that $\sum_{l=0}^{L-1} \delta_{l}^{2}=1$. In (1), the transmitted signal $x_{i}(n)$ is assumed to have the energy constraint $E \sum_{n=0}^{N-1} \sum_{i=1}^{M_{t}}\left|x_{i}(n)\right|^{2}=N$.

In the following, we propose a differential encoding/decoding scheme in the frequency domain within each single OFDM block, based on a space-frequency structure developed in [17] and [18]. For any fixed integer $\Gamma(1 \leq \Gamma \leq L)$, a class of space-frequency signals were designed in [17] and [18] that are guaranteed to achieve a diversity order of $\Gamma M_{t} M_{r}$ in a coherent scenario. Specifically, denote $P=\left\lfloor N /\left(\Gamma M_{t}\right)\right\rfloor$, which is the largest integer not greater than $N /\left(\Gamma M_{t}\right)$, and denote $B_{p}=(p-1) \Gamma M_{t}$ for any $p=1,2, \ldots, P$. We specify the transmitted signal $x_{i}(n)$ in (1) as follows: 1$) x_{i}(n) \neq 0$ for any $n=B_{p}+(i-1) \Gamma+\gamma-1$, in which $1 \leq p \leq P, 1 \leq i \leq M_{t}$, and $1 \leq \gamma \leq \Gamma$; and 2) $x_{i}(n)=0$ for other $n$. It means that at each subcarrier, there is only one transmit antenna that sends nonzero symbols. Then, the received signal in (1) can be written as $y_{j}(n)=\sqrt{\rho} x_{i}(n) H_{i, j}(n)+z_{j}(n)$ for any
$n=B_{p}+(i-1) \Gamma+\gamma-1$ with $1 \leq p \leq P, 1 \leq i \leq M_{t}$, and $1 \leq \gamma \leq \Gamma$. We further format the received signals in a compact matrix form as follows:

$$
\mathbf{Y}_{p}=\sqrt{\rho} \mathbf{X}_{p} \mathbf{H}_{p}+\mathbf{Z}_{p}, \quad p=1,2, \ldots, P
$$

where $\mathbf{X}_{p}=\operatorname{diag}\left(x_{i}\left(B_{p}+(i-1) \Gamma+\gamma-1\right): 1 \leq i \leq M_{t}\right.$, $1 \leq \gamma \leq \Gamma)$ is a $\Gamma M_{t} \times \Gamma M_{t}$ diagonal matrix. In (2), the received signal matrix $\mathbf{Y}_{p}$ is of size $\Gamma M_{t}$ by $M_{r}$, whose $(k, j)$ th $(1 \leq$ $\left.k \leq \Gamma M_{t}, 1 \leq j \leq M_{r}\right)$ entry is $y_{j}\left(B_{p}+k-1\right)$, the channel matrix $\mathbf{H}_{p}$ also has size $\Gamma M_{t}$ by $M_{r}$, whose $((i-1) \Gamma+\gamma, j)$ th entry is $H_{i, j}\left(B_{p}+(i-1) \Gamma+\gamma-1\right)$, and the noise matrix $\mathbf{Z}_{p}$ has the same format as $\mathbf{Y}_{p}$.

With the transceiver model in (2), we encode the transmitted signals differentially in the frequency dimension within each OFDM block as follows: $\mathbf{X}_{p}=V_{l_{p}} \mathbf{X}_{p-1}, p=2,3, \ldots, P$, where $\mathbf{X}_{1}=I_{\Gamma M_{t} \times \Gamma M_{t}}$, and $V_{l_{p}}$ carries the transmitted information and is a unitary diagonal matrix chosen from a cyclic signal constellation designed in [4]. We consider differential decoding over two received matrices $\mathbf{Y}_{p}$ and $\mathbf{Y}_{p-1}$ for any $p=$ $2,3, \ldots, P$ within each OFDM block as follows. Since $\mathbf{Y}_{p}=$ $\sqrt{\rho} V_{l_{p}} \mathbf{X}_{p-1} \mathbf{H}_{p}+\mathbf{Z}_{p}$, and $\mathbf{Y}_{p-1}=\sqrt{\rho} \mathbf{X}_{p-1} \mathbf{H}_{p-1}+\mathbf{Z}_{p-1}$, we have

$$
\mathbf{Y}_{p}=V_{l_{p}} \mathbf{Y}_{p-1}+\sqrt{\rho} \mathbf{X}_{p} \Delta_{p}+\mathbf{Z}_{p}^{\prime}
$$

where $\boldsymbol{\Delta}_{p}=\mathbf{H}_{p}-\mathbf{H}_{p-1}$ is the channel difference between $\mathbf{H}_{p}$ and $\mathbf{H}_{p-1}$, and $\mathbf{Z}_{p}^{\prime}=\mathbf{Z}_{p}-V_{l_{p}} \mathbf{Z}_{p-1}$ is a noise matrix where each entry is a Gaussian random variable with mean zero and variance $\sqrt{2}$, since $V_{l_{p}}$ is unitary. If $\mathbf{H}_{p} \approx \mathbf{H}_{p-1}$, or the Frobenius norm of the channel difference $\left\|\boldsymbol{\Delta}_{p}\right\|_{F}$ is small enough such that $\sqrt{\rho}\left\|\Delta_{p}\right\|_{F}$ is much less than $\left\|\mathbf{Z}_{p}^{\prime}\right\|_{F}$, then a near-maximum-likelihood (ML) decoding ${ }^{1}$ can be performed as

$$
\hat{l_{p}}=\arg \min _{0 \leq l_{p} \leq L_{0}-1}\left\|\mathbf{Y}_{p}-V_{l_{p}} \mathbf{Y}_{p-1}\right\|_{F} .
$$

In case the delay spread is large with respect to the OFDM period, it is hard to assume that the changing of the channel frequency responses is slow over two adjacent subcarriers. However, if a statistical channel PDP is known at the transmitter, we are able to permute the transmitted signals over different subcarriers to create a smooth logical channel. Denote the permuted channel as $\tilde{\mathbf{H}}_{p}$, and accordingly denote the received signal matrix and the noise matrix as $\tilde{\mathbf{Y}}_{p}$ and $\tilde{\mathbf{Z}}_{p}^{\prime}$, respectively. Similar to (3), we have $\tilde{\mathbf{Y}}_{p}=V_{l_{p}} \tilde{\mathbf{Y}}_{p-1}+\sqrt{\rho} \mathbf{X}_{p} \tilde{\boldsymbol{\Delta}}_{p}+\tilde{\mathbf{Z}}_{p}^{\prime}$, where $\tilde{\boldsymbol{\Delta}}_{p}=\tilde{\mathbf{H}}_{p}-\tilde{\mathbf{H}}_{p-1}$ and $\tilde{\mathbf{Z}}_{p}^{\prime}=\tilde{\mathbf{Z}}_{p}-V_{l_{p}} \tilde{\mathbf{Z}}_{p-1}$. The decoding can be performed accordingly as $\hat{l_{p}}=\arg \min _{0 \leq l_{p} \leq L_{0}-1} \| \tilde{\mathbf{Y}}_{p}-$ $V_{l_{p}} \tilde{\mathbf{Y}}_{p-1} \|_{F}$.

\section{OBtaining SMOOTH LOGICAL CHANNELS FOR THE DSFM SCHEME}

Assume in this section that a statistical channel PDP (not exact CSI) is known at the transmitter. We want to obtain a smooth logical channel by permutations, such that the differential decoding can perform well even if the channel frequency responses may vary severely over two adjacent subcarriers.

\footnotetext{
${ }^{1}$ Note that the differential decoding in (4) is ML only if $\mathbf{H}_{p}=\mathbf{H}_{p-1}$. Here we term it as a near-ML decoding in the sense that we try to sort the channel such that $\mathbf{H}_{p} \approx \mathbf{H}_{p-1}$.
} 
From the system performance point of view, one should optimize the permutations by maximizing the bit-error rate (BER). However, this approach is intractable, if not impossible. We consider an approach of maximizing the average signal-to-noise ratio (SNR), which is defined as

$$
\mathrm{SNR}_{\text {average }}=\frac{\sum_{p=2}^{P}\left(E\left\|V_{l_{p}} \tilde{\mathbf{Y}}_{p-1}\right\|_{F}^{2}\right)}{\sum_{p=2}^{P}\left(E\left\|\sqrt{\rho} \mathbf{X}_{p} \tilde{\Delta}_{p}+\tilde{\mathbf{Z}}_{p}^{\prime}\right\|_{F}^{2}\right)}
$$

Note that both $V_{l_{p}}$ and $\mathbf{X}_{p}$ are unitary matrices, so $E\left\|V_{l_{p}} \tilde{\mathbf{Y}}_{p-1}\right\|_{F}^{2}=(\rho+1) \Gamma M_{t} M_{r}$, and $E \| \sqrt{\rho} \mathbf{X}_{p} \tilde{\Delta}_{p}+$ $\tilde{\mathbf{Z}}_{p}^{\prime}\left\|_{F}^{2}=\rho E\right\| \tilde{\boldsymbol{\Delta}}_{p} \|_{F}^{2}+2 \Gamma M_{t} M_{r}$. Therefore, $\mathrm{SNR}_{\text {average }}=$ $(\rho+1) /(\rho \Phi+2)$, in which $\Phi$ is an average channel mismatch

$$
\Phi=\frac{1}{(P-1) \Gamma M_{t} M_{r}} \sum_{p=2}^{P} E\left\|\tilde{\Delta}_{p}\right\|_{F}^{2} .
$$

In order to maximize the average SNR, we try to minimize the average channel mismatch $\Phi$ in (6). For simplicity, denote $n_{p, k}=B_{p}+k$ for any $2 \leq p \leq P$ and $0 \leq k \leq \Gamma M_{t}-1$. We assume that with permutation, the $n_{p, k}$ th subcarrier of the original channel is permuted to the $\sigma\left(n_{p, k}\right)$ th subcarrier. Since the fading channels between different transmit and receive antenna pairs are assumed to have the same PDP, we can determine $E\left\|\tilde{\Delta}_{p}\right\|_{F}^{2}$ for any $1 \leq p \leq P$ as follows:

$$
\begin{aligned}
E\left\|\tilde{\boldsymbol{\Delta}}_{p}\right\|_{F}^{2}= & M_{r} \sum_{k=0}^{\Gamma M_{t}-1} \sum_{l=0}^{L-1} E\left|\alpha_{1,1}(l)\right|^{2} \\
& \times\left|e^{-\frac{\mathbf{j} 2 \pi \sigma\left(n_{p, k}\right) \tau_{l}}{T}}-e^{-\frac{\mathbf{j} 2 \pi \sigma\left(n_{p-1, k}\right) \tau_{l}}{T}}\right|^{2} \\
= & M_{r} \sum_{k=0}^{\Gamma M_{t}-1} \sum_{l=0}^{L-1} d\left(\sigma\left(n_{p, k}\right), \sigma\left(n_{p-1, k}\right)\right)
\end{aligned}
$$

where $d(i, j)=\sum_{l=0}^{L-1} 4 \sin ^{2}\left[\pi(i-j) \tau_{l} / T\right] \delta_{l}^{2}$. Then, the average channel mismatch $\Phi$ in (6) is

$$
\Phi=\frac{1}{(P-1) \Gamma M_{t}} \sum_{p=2}^{P} \sum_{k=0}^{\Gamma M_{t}-1} d\left(\sigma\left(n_{p, k}\right), \sigma\left(n_{p-1, k}\right)\right) .
$$

The minimization of the channel mismatch $\Phi$ can be reduced to the shortest-path problem [20]. To illustrate the basic idea, we first consider a special case where $M_{t}=1$ and $\Gamma=1$, i.e., $P=N$. Let us think about a network with $N$ nodes, in which the distance between node $i$ and node $j(1 \leq i, j \leq N)$ is $d(i, j) /(N-1)$. Then for any permutation, the channel mismatch $\Phi$ is the length of a path that goes over all nodes in the network. Therefore, the problem of minimizing the channel mismatch $\Phi$ is reduced to the shortest-path problem [20]. The shortest-path problem can be solved by using Dijkstra's algorithm [19], [20], shown as follows.

- $\quad$ Let $V_{0}=\{1,2, \ldots, N\}$ denote the network with all nodes. Start from node 1 by setting $e_{1}=1$ and denoting the set of the rest nodes as $V_{1}=V_{0}-\{1\}$.
- $\quad$ For $i=2: N$

1) Find a node $j \in V_{i-1}$ which is nearest to node $i-1$, i.e., to choose a node $j$ in $V_{i-1}$ such that the distance $d(i-1, j)$ is minimized.

2) Denote $e_{i}=j$ and let the set of the rest nodes be $V_{i}=$ $V_{i-1}-\{j\}$.

The above algorithm results in a shortest path that starts at node 1 and goes over all other nodes which shows a desired permutation that minimizes the channel mismatch $\Phi$.

For arbitrary $\Gamma M_{t} \geq 2$, let us consider a network with nodes $\{1,2, \ldots, P\}$, in which each node stands for a set that contains $\Gamma M_{t}$ elements. The distance between two nodes or sets $p$ and $q(1 \leq p, q \leq P)$ is assumed to be $D(p, q)=\left(1 / \Gamma M_{t}\right) \sum_{k=0}^{\Gamma M_{t}-1} d\left(n_{p, k}, n_{q, k}\right)$. Then, the channel mismatch $\Phi$ in (7) can be considered as the length of a "path" that goes over all $P$ sets in the network. In order to find a shortest "path" going over all sets, we propose a generalized Dijkstra's algorithm as follows.

- $\quad$ Let $V_{0}=\left\{1,2, \ldots, P \Gamma M_{t}\right\}$. Randomly choose $\Gamma M_{t}$ elements $\left\{n_{1,1}, n_{1,2}, \ldots, n_{1, \Gamma M_{t}}\right\}$ from $V_{0}$ to form a set $E_{1}$, and let the set of the rest elements be $V_{1}=V_{0}-E_{1}$.

- $\quad$ For $p=2: P$

1) Choose $\Gamma M_{t}$ elements $\left\{n_{p, 1}, \ldots, n_{p, \Gamma M_{t}}\right\}$ from $V_{p-1}$, to form a set $E_{p}$ whose elements are nearest to the set $E_{p-1}$, i.e., to minimize the distance $D(p, p-1)$.

2) Denote the set of the rest elements as $V_{i}=V_{i-1}-E_{p}$.

An interesting question is, how smooth a logical channel can we achieve? Let us define a minimum channel mismatch $\Delta_{0}=$ $\min _{0 \leq m<n \leq N-1} \sum_{l=0}^{L-1} 4 \sin ^{2}\left[\pi(m-n) \tau_{l} / T\right] \delta_{l}^{2}$. With any permutation, it is easy to see that the channel mismatch $\Phi$ in (7) is lower bounded by $\Delta_{0}$, i.e., $\Phi \geq \Delta_{0}$. For some channel PDPs, such as the two-ray fading model, we are able to obtain smooth logical channels such that the resulting channel mismatch $\Phi$ can reach $\Delta_{0}$. However, for others, such as the typical urban (TU) model [21], we are not able to obtain smooth logical channels such that $\Phi$ can reach the minimum channel mismatch $\Delta_{0}$. However, in this case, we found that with the generalized Dijkstra algorithm, some of the channel mismatches $D(p, q)$ are larger than the minimum mismatch $\Delta_{0}$, and some of them can reach $\Delta_{0}$.

The above observation motivates us to propose a pruning process, as follows. We do not use the "bad" part of the channel whose channel mismatch $D(p, q)$ is larger than $\Delta_{0}$, and use only the "good" part of the channel whose channel mismatch $D(p, q)$ is equal to $\Delta_{0}$. With such a pruning process, the DSFM scheme may perform successfully over the "good" part of the logical channel, if the minimum channel mismatch $\Delta_{0}$ is small. Since we use only a part of the channel, the spectral efficiency of the system is reduced. Suppose that we prune $N_{1}$ subchannels and use only $N_{2}$ subchannels in the logical channel, then the spectral efficiency is reduced by a factor of $N_{1} /\left(N_{1}+N_{2}\right)$. As shown in the simulation results in the next section, if we use all of the channel, there is a high error floor in the performance curve, due to the "bad" part of the channel. By use of the pruning process over the logical channel, the channel mismatch in the remaining "good" part of the channel reaches the minimum channel mismatch. As a consequence, the performance 


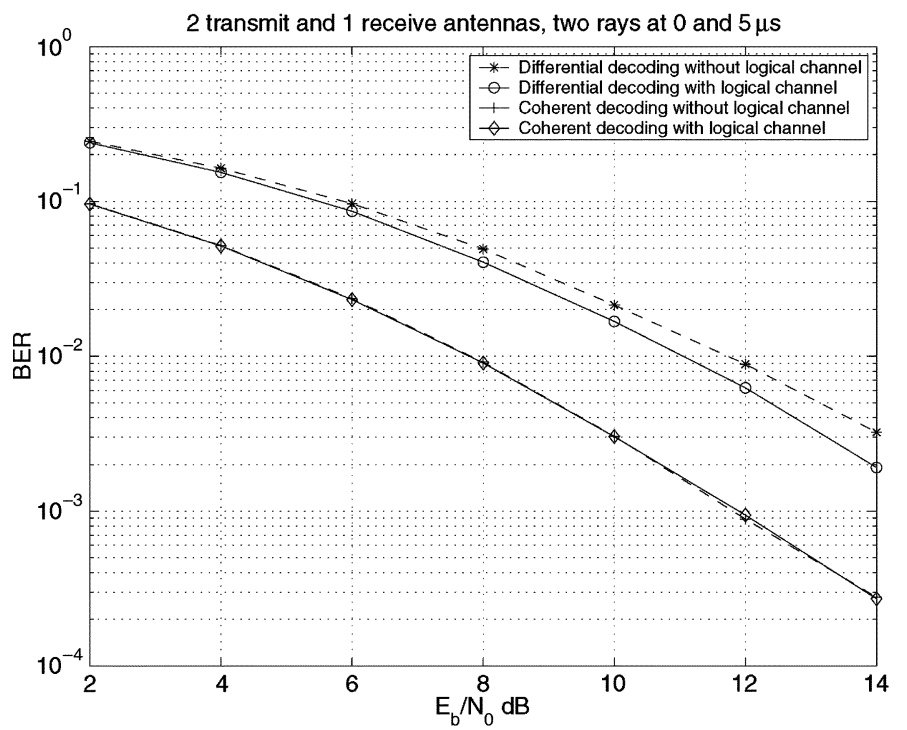

Fig. 1. Performances of the DSFM scheme with the two-ray channel model in the case of $\tau=5 \mu \mathrm{s}$.

of the DSFM scheme over the resulting channel depends only on the minimum channel mismatch. Note that if the minimum channel mismatch is too large, then we have no chance to obtain a smooth logical channel even with the pruning process.

\section{Simulation Results}

We simulated the proposed DSFM scheme for a system with $M_{t}=2$ transmit and $M_{r}=1$ receive antennas. The OFDM modulation had $N=128$ subcarriers. The used cyclic signal constellation is $\left\{V_{l}=\operatorname{diag}\left(e^{\mathbf{j} \pi l / 16}, e^{\mathbf{j} 3 \pi l / 16}, e^{\mathbf{j} 5 \pi l / 16}\right.\right.$, $\left.\left.e^{\mathrm{j} 7 \pi l / 16}\right), l=0,1, \ldots, 15\right\}$, in which each signal matrix $V_{l}$ represents an information symbol of 4 bits [4].

At first, we considered a two-ray $\operatorname{PDP}(L=2)$, with a delay of $\tau \mu$ s between the two rays. Each ray was modeled as a zeromean complex Gaussian random variable with variance 0.5 . We simulated two cases: 1) $\tau=5 \mu$ s and 2) $\tau=20 \mu$ s. Assume that the total bandwidth was $1 \mathrm{MHz}$. Then, the OFDM block duration was $T=128 \mu$ s without the cyclic prefix. The performance of the proposed DSFM scheme is shown in Fig. 1 for the case of $\tau=5 \mu$ s. Since the $5 \mu$ s separation of the two paths is small compared with the $128 \mu$ s duration of the OFDM block, the channel frequency responses change smoothly over different subcarriers. We can see that the DSFM scheme performs successfully in this case, and the performance of the scheme with a smooth logical channel is a little better than that without a smooth channel. In case of $\tau=20 \mu \mathrm{s}$, there are severe variations in the spectrum of the channel impulse responses, resulting in the failure of the DSFM scheme without channel permutation, as shown in Fig. 2. However, if we permute the channel properly, we are able to obtain a smooth logical channel that can guarantee the successful differential decoding, as shown in the solid curve with circles.

We also simulated the proposed DSFM scheme over the TU fading model [21] with three different bandwidths: 1) BW $=1$ MHz; 2) $\mathrm{BW}=4 \mathrm{MHz}$; and 3) $\mathrm{BW}=20 \mathrm{MHz}$, as shown in Fig. 3. In the case of $\mathrm{BW}=1 \mathrm{MHz}$, the OFDM duration was

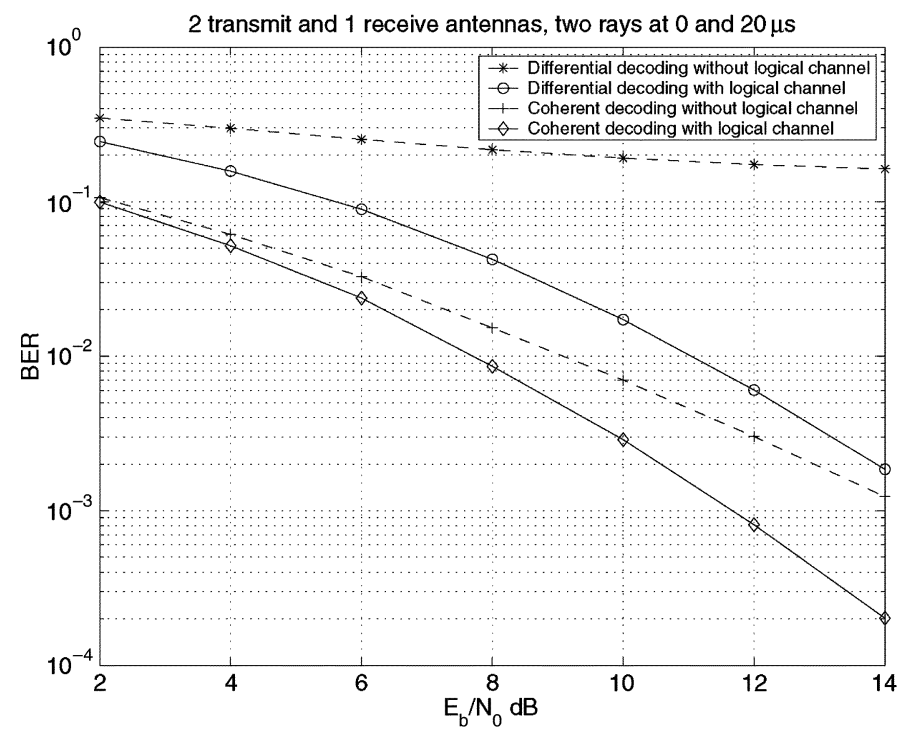

Fig. 2. Performances of the DSFM scheme with the two-ray channel model in the case of $\tau=20 \mu \mathrm{s}$.

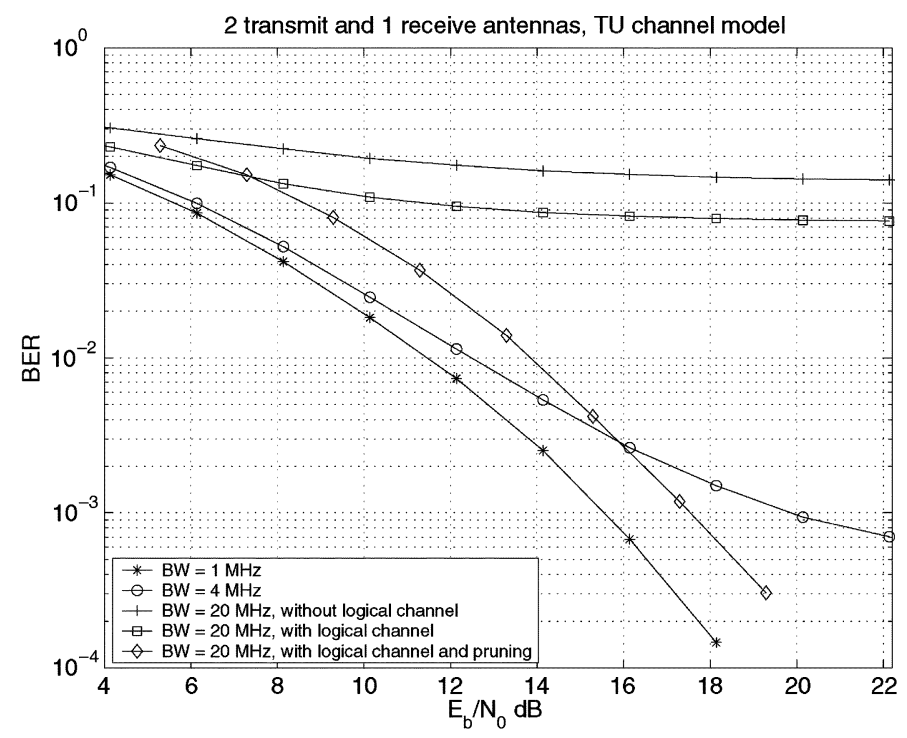

Fig. 3. Performances of the DSFM scheme with the TU channel model.

$T=128 \mu$ s. Since the delay spread of the TU fading model is small, compared with the $128 \mu$ s duration of the OFDM block, the channel frequency responses change smoothly over different subcarriers. We can see that the DSFM scheme performed successfully over this channel. In the case of BW $=4 \mathrm{MHz}$, the OFDM duration was $T=32 \mu \mathrm{s}$. From the performance curve (with "o"), we observe that the DSFM scheme performed well at low SNR, but had an error floor at high SNR due to a large minimum channel mismatch. In the case of $\mathrm{BW}=20 \mathrm{MHz}$, the OFDM duration was $T=6.4 \mu \mathrm{s}$. Since the delay spread of the TU fading model is not small, compared with the $6.4 \mu$ s duration of the OFDM block, there are severe variations in the spectrum of the channel impulse responses. From the performance curve (with "+"), we can see that the severe variations of the channel result in the failure of the DSFM scheme, as we applied the DSFM scheme directly. With such a channel, even though we used the generalized Dijkstra algorithm to sort the channel, the 
resulting logical channel was still not applicable, and the performance curve (with " $\square$ ") has a very bad error floor. We found that in the logical channel, some of the channel mismatches $D(p, q)$ are 0.5200 , and the others are 0 . The minimum channel mismatch $\Delta_{0}$ is zero in this case. Therefore, we pruned the part of channel with mismatches $D(p, q)=0.5200$, and used only the part of channel with mismatches $D(p, q)=\Delta_{0}$. From the performance curve (with " $\diamond$ "), we can see that the DSFM scheme performed very well over the logical channel with pruning.

\section{CONCLUSION}

In this letter, a differential MIMO-OFDM modulation scheme was proposed for broadband wireless communications, in which the transmitted signals were differentially encoded across multiple antennas in the frequency domain within each single OFDM block. Thus, the proposed DSFM scheme can be applied over the scenarios where the fading channel may change from one OFDM block to another independently. Moreover, if a statistical channel PDP is known at the transmitter, we further proposed to permute the channel frequency responses over subcarriers to create a smooth logical channel, by using the generalized Dijkstra algorithm. For some channel PDPs where there are severe variations in the spectrum of the channel impulse responses, and even the generalized Dijkstra algorithm may not work well, we proposed in this case a pruning process to get rid of the "bad" part of the channel. Simulation results showed that in case of the TU channel model with BW $=20$ $\mathrm{MHz}$, the DSFM scheme performed very well over the logical channel with pruning.

\section{REFERENCES}

[1] E. Telatar, "Capacity of multi-antenna Gaussian channels,", AT\&T Bell Labs, Tech. Rep., Jun. 1995.

[2] V. Tarokh and H. Jafarkhani, "A differential detection scheme for transmit diversity," IEEE J. Sel. Areas Commun., vol. 18, no. 7, pp. 1169-1174, Jul. 2000.

[3] B. L. Hughes, "Differential space-time modulation," IEEE Trans. Inf. Theory, vol. 46, no. 11, pp. 2567-2578, Nov. 2000.
[4] B. M. Hochwald and W. Sweldens, "Differential unitary space-time modulation," IEEE Trans. Commun., vol. 48, no. 12, pp. 2041-2052, Dec. 2000

[5] A. Shokrollahi, B. Hassibi, B. H. Hochwald, and W. Sweldens, "Representation theory for high-rate multiple-antenna code design," IEEE Trans. Inf. Theory, vol. 47, no. 6, pp. 2335-2367, Jun. 2001.

[6] B. Hassibi and B. M. Hochwald, "Cayley differential unitary space-time codes," IEEE Trans. Inf. Theory, vol. 48, no. 6, pp. 1485-1503, Jun. 2002.

[7] B. L. Hughes, "Optimal space-time constellations from groups," IEEE Trans. Inf. Theory, vol. 49, no. 2, pp. 401-410, Feb. 2003.

[8] C. Shan, A. Nallanathan, and P. Y. Kam, "A new class of signal constellations for differential unitary space-time modulation (DUSTM)," IEEE Commun. Lett., vol. 8, no. 1, pp. 1-3, Jan. 2004.

[9] H. Bolcskei and M. Borgmann, "Code design for noncoherent MIMO-OFDM systems," in Proc. 40th Allerton Conf. Commun., Control, Comput., Monticello, IL, Oct. 2002, pp. 237-246.

[10] — , "Non-coherent space-frequency coded MIMO-OFDM," IEEE J. Sel. Areas Commun., vol. 23, no. 9, pp. 1799-1810, Sep. 2005.

[11] S. N. Diggavi, N. Al-Dhahir, A. Stamoulis, and A. R. Calderbank, "Differential space-time coding for frequency-selective channels," IEEE Commun. Lett., vol. 6, no. 6, pp. 253-255, Jun. 2002.

[12] Z. Liu and G. B. Giannakis, "Block differentially encoded OFDM with maximum multipath diversity," IEEE Trans. Wireless Commun., vol. 2, no. 3, pp. 420-423, May 2003.

[13] Q. Ma, C. Tepedelenlioglu, and Z. Liu, "Differential space-time-frequency coded OFDM with maximum diversity," in Proc. 37th Annu. Conf. Inf. Sci. Syst., Baltimore, MD, Mar. 2003, pp. 121-126.

[14] —, "Full diversity block diagonal codes for differential space-time-frequency coded OFDM," in Proc. IEEE GLOBECOM, vol. 2, 2003, pp. 868-872.

[15] H. Li, "Differential space-time modulation with maximum spatio-spectral diversity," in Proc. IEEE Int. Conf. Commun., vol. 4, 2003, pp. $2588-2592$.

[16] M. Lott, "Comparison of frequency and time domain differential modulation in an OFDM system for wireless ATM," in Proc. IEEE 49th Veh. Technol. Conf., vol. 2, May 1999, pp. 877-883.

[17] W. Su, Z. Safar, and K. J. R. Liu, "Systematic design of space-frequency codes with full rates and full diversity," in Proc. IEEE Wireless Commun. Netw. Conf., vol. 3, Atlanta, GA, Mar. 2004, pp. 1436-1441.

[18] — "Full-rate full-diversity space-frequency codes with optimum coding advantage," IEEE Trans. Inf. Theory, vol. 51, no. 1, pp. 229-249, Jan. 2005.

[19] E. L. Lawler, J. K. Lenstra, and A. H. G. Rinnooy Kan, The Traveling Salesman Problem. New York: Wiley, 1983.

[20] G. Gallo and S. Pallotino, "Shortest path methods: A unifying approach," Math. Program. Study, vol. 26, pp. 38-64, 1986.

[21] G. Stuber, Principles of Mobile Communication. Norwell, MA: Kluwer, 2001. 